\title{
A novel heterozygous TPM2 gene mutation (c.456G >C; p.Lys152Asn) in an Iranian family affected by distal arthrogryposis type 1: a case report
}

\author{
Mostafa Neissi ${ }^{1,2^{*}} \mathbb{C}$, Motahareh Sheikh-Hosseini ${ }^{3}$, Javad Mohammadi-As| ${ }^{4}$ and Adnan Issa Al-Badran ${ }^{5}$
}

\begin{abstract}
Background: Distal arthrogryposis (DA) is a group of clinically and genetically heterogeneous disorders characterized by congenital contractures of the distal limb joints without a neuromuscular disease. This case study seeks to report an affected son from an Iranian family with a diagnosis of DA1 with a rare causative genetic defect.

Case presentation: Herein, we aimed to figure out the underlying genetic of the subject from an Iranian family with DA1. Whole-exome sequencing (WES) of all known DA1 genes was carried out in the proband of the family. WES identified a novel missense mutation, c.456G>C; p.Lys152Asn, within the TPM2 gene, causing a change in one amino acid (Lysine converted to Asparagine). Moreover, this detected variant was confirmed by Sanger sequencing.

Conclusion: Our data expand the mutational spectra of TPM2 gene associated DA1 which is vital for screening and genetic diagnosis of the disease. Also, this detected mutation has not yet been described in patients with the DA1 phenotype.
\end{abstract}

Keywords: TPM2 gene, Distal arthrogryposis type 1, Mutation

\section{Background}

The distal arthrogryposes (DA) are a group of autosomaldominant disorders affecting primarily the distal parts of the limbs. The distal arthrogryposes result from congenital contractures of two or more body parts, without any underlying neurological and/or muscular disease. Approximately 1 in every 3000 live births is affected by arthrogryposis [1].

Clinically and genetically, DA is heterogeneous and comprises more than ten different clinical subtypes (1, 2A, 2B, 3-10). DA1 (OMIM 108120) and DA2B (OMIM 601680 ) are the most common subtypes. Camptodactyly and clubfoot are the most prominent features of DA1, but

\footnotetext{
*Correspondence: iammostafaneissi@gmail.com

1 Department of Genetics, Khuzestan Science and Research Branch,

Islamic Azad University, Ahvaz, Iran

Full list of author information is available at the end of the article
}

the hallmarks of DA2B include downward slanting, palpebral fissures, small mouth, triangular face, prominent nasolabial folds, attached earlobes, prominent chin, and severe camptodactyly $[1,2]$.

To date, 8 genes have been reported to cause DA, including TPM2, MYBPC1, TNNI2, TNNT3, MYH3, ECEL1, MYH8, and FBN2. Among these genes, TPM2 gene is a significant disease-causing gene of DA1 clinical manifestations [3, 4].

Though almost all disease-causing TPM2 gene mutations are inherited in an autosomal-dominant manner, one homozygous nonsense TPM2 gene mutation was detected in a patient with a very severe nemaline myopathy. Whole-exome sequencing (WES) can be applied as a useful diagnostic method to identify disease-causing mutations in these cases [5]. 
In the present study, we employed WES and Sanger sequencing to discover the causative genetic defect in an Iranian family affected by DA1. We recognized a novel pathogenic mutation of TPM2 gene that could explain the DA1 phenotype in this family.

\section{Case presentation}

We collected one DA1 family who came to the Noor Gene Genetic lab, Ahvaz, Iran (Fig. 1). The proband, a 5 -year-old boy was diagnosed with DA1. Based on clinical evaluations, the proband had congenital ulnar bilateral and symmetric arthrogryposes of 5th fingers without other malformation. In addition, this family reported that I:1, II:2, II:5, II:8, III:3, and III:6 had DA, but I:2, II:1, II:3, II:4, II:6, II:7, II:9, II:10, II:11, II:12, III:1, III:2, III:4, III:5, III:7, III:8, III:9, and IV:1 were phenotypically normal. Written informed consent was obtained from all participants, and the study was carried out according to the guidelines of the Declaration of Helsinki.

Peripheral blood samples were collected from the patient and other members of his family, and genomic DNA was isolated from blood samples of all participants by using standard salting-out protocol. NanoDrop 1000 spectrophotometer was used to measure the concentration of DNA samples.

Next, WES was only performed on the proband to find out the pathogenic variant. The DNA sequence analysis of the genes involved in the pathogenesis of DA1 showed a heterozygous mutation in TPM2 gene and no other potential disease-causing variants were identified. This mutation is a novel and rare missense heterozygous mutation (c.456G>C), located in exon 4 (NM_003289.4) and results in substitution of Lysine to Asparagine at codon 152.

The international publicly available mutation and polymorphism databases (1000 Genomes Project, ExAC browser) were used for the analysis and interpretation of sequencing data. Only low-frequency of detected variants (less than 1\%) were selected. This variant of uncertain significance has not been reported. Three bioinformatics tools for analyzing protein functions, SIFT, PolyPhen2, and MutationTaster, predicted that $\mathrm{K} 152 \mathrm{~N}$ variant is probably damaging, deleterious, and disease-causing.

Sanger sequencing was carried out in the patient and family members to confirm the true positive of the identified TPM2 variant. The genomic DNA samples were amplified by polymerase chain reaction (PCR) and then the PCR products were directly sequenced on the automated genetic analyzer (ABI3130, Applied Biosystems, USA). The results showed heterozygous change in the proband and his affected father, but the normal members in the family were lack of K152N mutation (Fig. 2A). The alignment of TPM2 from seven diverse species is shown in Fig. 2B. This result revealed that this variant occurred in a conserved region of the protein. Therefore, it is recommended that p.K152N residue is important for protein organization and function.

\section{Discussion}

Common birth defects like congenital contractures are related to significant morbidity and economic burden. Understanding the etiology and pathogenesis of congenital contractures is an essential aspect of research in pediatric health care [1]. Thus, in this study, for a better

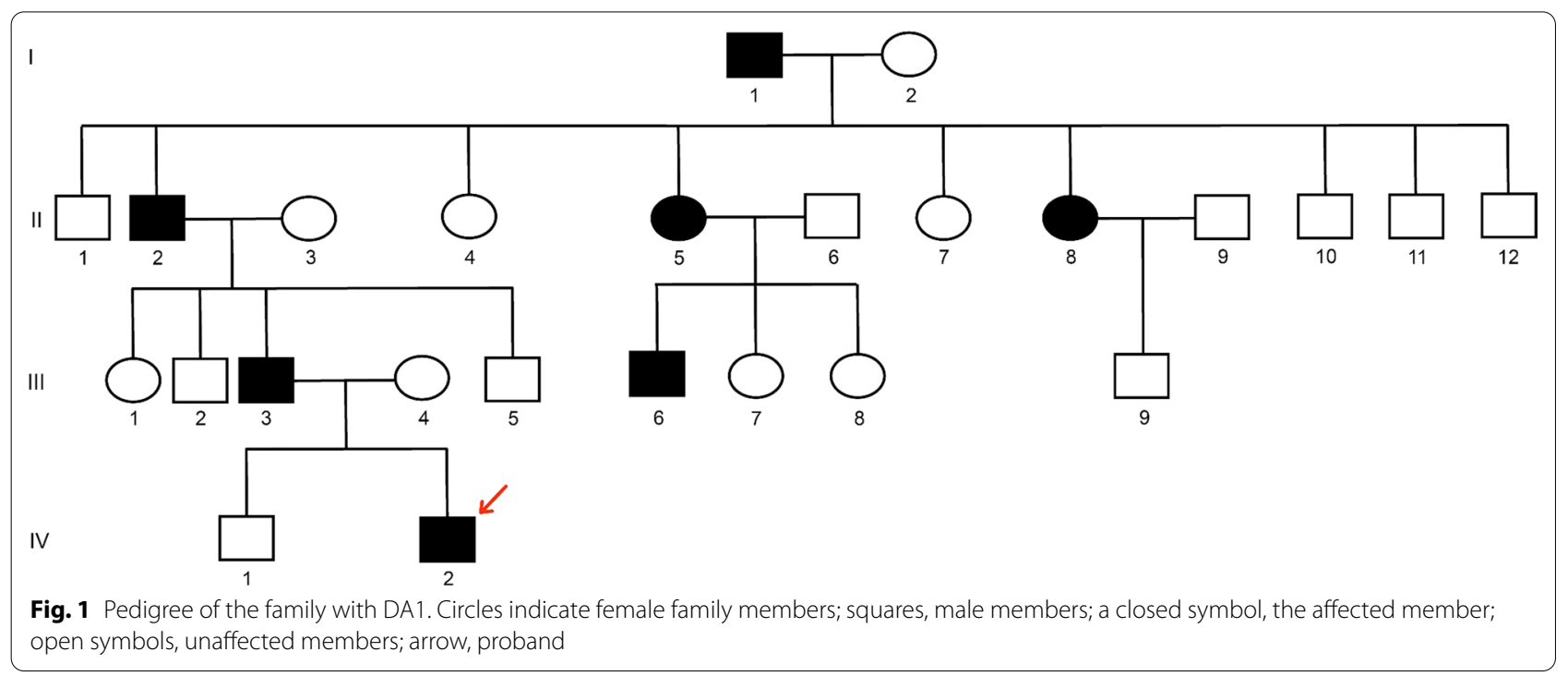




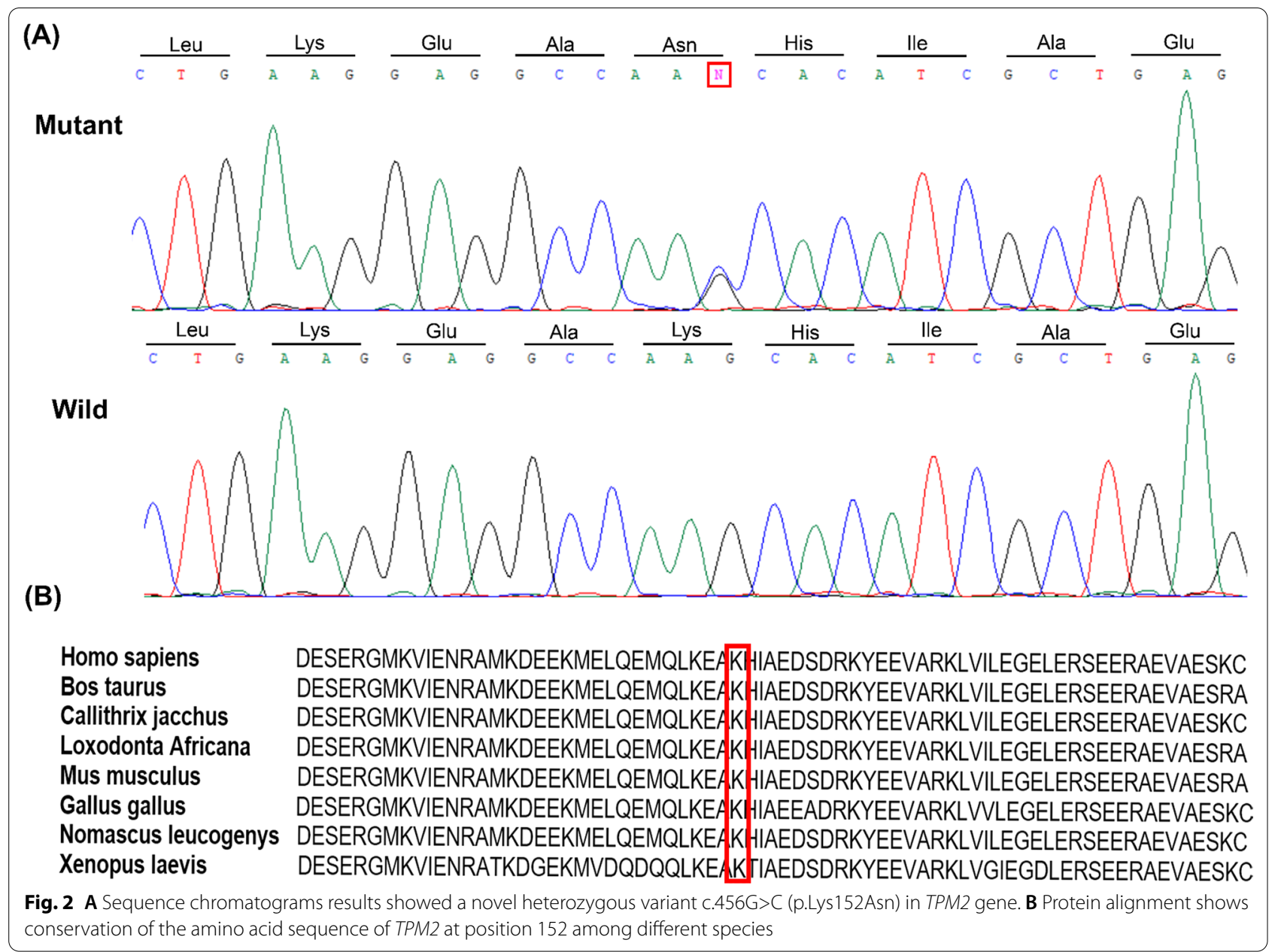

understanding of the molecular genetics causes of the DA1 disorder in the family, the WES technique was used.

The Iranian family described here has DA1 with autosomal-dominant inheritance, WES found a novel heterozygous mutation in TPM2 gene to be the underlying causes of the disorder in the family.

TPM2 gene encodes skeletal muscle isoforms TPM2 and belongs to the tropomyosin gene family (including TPM1-4). There are 284 amino acid residues in this isoform, and a highly conserved $\mathrm{N}$-terminal region. TPM2 gene is a major cause of congenital myopathies, and at least 35 mutations have been reported. These mutations can lead to different phenotypes, such as DA1, DA2B, nemaline myopathy (NM), congenital fiber type disproportion (CFTD), and cap disease [4].

In one study, Anita E. Beck et al. evaluated 48 children with DA1 for TNNI2, TPM2, TNNT3, and MYH3 mutations. Their results showed a frequency of 29\% (14/48) of all patient carried a mutation in these genes that cause DA1 and with only three mutations (c.271C>G; p.Arg91Gly, c.289G>A; p.Glu97Lys, and c.397C>T;
p.Arg133Trp) in the TPM2 gene [6]. In another investigation, Jie-Yuan Jin et al. revealed that a heterozygous c.463G >A; p.Ala155Thr mutation in TPM2 gene is correlated with DA1 clinical manifestations [4]. In our study, we also report a new heterozygous missense mutation (c.456G>C; p.Lys152Asn) that is related to DA1.

It was demonstrated that most patients affected with DA1 exhibit flexion contractures at birth. The hands and feet are the most commonly affected joints. The hands are typically held in a characteristic position consisting of a tight fist with medial overlapping of the fingers and an adducted thumb [7]. Furthermore, other studies concluded that this form of DA involving TPM2 mutation is accompanied by arthrogryposis of fingers $[4,8]$ and this finding is consistent with our results.

To the best of our knowledge, this mutation has not previously been reported in any of the mutation databases. The following evidence proves that this mutation can lead to DA1: 1- Only this mutation was identified as the cause of DA1 by WES. 2- As can be seen in Fig. 2, direct Sanger sequencing proved the mutation in the 
proband and his affected father, and based on detected heterozygote mutations in them, the pattern of inheritance for TPM2 gene must be autosomal-dominant. 3- Bioinformatic tools predicted that this variant to be pathogenic. 4- We believe that the TPM2-p.K152N mutation is the pathogenic variant for DA1 because the mutated Lysine amino acid is evolutionarily conserved (Fig. 2B). 5- A missense mutation in exon 4 of TPM2 gene (c.456G>C; p.Lys152Asn), predicting an alteration in codon translation, and finally defects in function or stability. 6- On the other hand, because this modify was absent in the healthy members, it cannot be ruled out that it affects the phenotype of this patient. Thus, this TPM2 gene mutation is pathogenic in our patient with DA1.

Finally, this detected mutation seems to be a rare mutation that inherited from patient ancestors. Based on our results, WES is a very valuable method for the detection of probable or novel mutations causing DA1.

\section{Conclusion}

In the present study, we have successfully applied WES for mutation screening within DA1 related genes and identified a rare novel mutation heterozygous missense TPM2 gene mutation (c.456G>C; p.Lys152Asn) in an Iranian pedigree. In addition, our data expand the spectrum of pathogenic mutations known to cause DA1.

\section{Abbreviations \\ CFTD: Congenital fiber type disproportion; DA: Distal arthrogryposes; NM: Nemaline myopathy; WES: Whole-exome sequencing.}

\section{Acknowledgements}

The authors would like to thank the family members for their participation in this study.

\section{Authors' contributions}

MN, MSH and JMA analyzed and interpreted the data. MN wrote the manuscript. MSH edited the manuscript. AlA helped to review the manuscript. All authors read and approved the final manuscript.

\section{Funding}

None.

\section{Availability of data and materials}

The data that support the findings of this study are available from the corresponding author upon reasonable request.

\section{Declarations}

\section{Ethics approval and consent to participate}

All procedures performed in this study were in accordance with the ethical standards of the institutional and/or national research committee and with the 1964 Helsinki declaration and its later amendments or compare ethical strand.

\section{Consent for publication}

Written informed consent was obtained from the family for this publication.

\section{Competing interests}

The authors declare that they have no competing interests.

\section{Author details}

${ }^{1}$ Department of Genetics, Khuzestan Science and Research Branch, Islamic Azad University, Ahvaz, Iran. ${ }^{2}$ Department of Genetics, Ahvaz Branch, Islamic Azad University, Ahvaz, Iran. ${ }^{3}$ Department of Medical Genetics, Institute of Medical Biotechnology, National Institute of Genetic Engineering and Biotechnology (NIGEB), Tehran, Iran. ${ }^{4}$ Noor Gene Genetic Lab, Ahvaz, Iran.

${ }^{5}$ Department of Biology, College of Science, University of Basrah, Basrah, Iraq.

Received: 11 November 2021 Accepted: 18 February 2022

Published online: 05 March 2022

\section{References}

1. Bamshad M, Van Heest AE, Pleasure D (2009) Arthrogryposis: a review and update. J Bone Joint Surg Am 91(Suppl 4):40

2. Li S, You Y, Gao J, Mao B, Cao Y, Zhao X et al (2018) Novel mutations in TPM2 and PIEZO2 are responsible for distal arthrogryposis (DA) 2B and mild DA in two Chinese families. BMC Med Genet 19(1):1-11

3. Daly SB, Shah H, O'sullivan J, Anderson B, Bhaskar S, Williams S et al (2014) Exome sequencing identifies a dominant TNNT3 mutation in a large family with distal Arthrogryposis. Mol Syndromol 5(5):218-228

4. Jin J-Y, Wu P-F, Fan L-L, YU F, Li J-J, Fan X-F et al (2017) A mutation of betatropomyosin gene in a Chinese family with distal arthrogryposis type I. Int J Clin Exp Pathol 10(11):11137

5. Mroczek M, Kabzińska D, Chrzanowska KH, Pronicki M, Kochański A (2017) A novel TPM2 gene splice-site mutation causes severe congenital myopathy with arthrogryposis and dysmorphic features. J Appl Genet 58(2):199-203

6. Beck AE, McMillin MJ, Gildersleeve HI, Kezele PR, Shively KM, Carey JC et al (2013) Spectrum of mutations that cause distal arthrogryposis types 1 and 2B. Am J Med Genet A 161(3):550-555

7. Bamshad M, Bohnsack JF, Jorde LB, Carey JC (1996) Distal arthrogryposis type 1: clinical analysis of a large kindred. Am J Med Genet 65(4):282-285

8. Kimber E, Tajsharghi H, Kroksmark AK, Oldfors A, Tulinius M (2012) Distal arthrogryposis: clinical and genetic findings. Acta Paediatr 101(8):877-887

\section{Publisher's Note}

Springer Nature remains neutral with regard to jurisdictional claims in published maps and institutional affiliations.

\section{Submit your manuscript to a SpringerOpen ${ }^{\odot}$ journal and benefit from:}

- Convenient online submission

- Rigorous peer review

- Open access: articles freely available online

- High visibility within the field

- Retaining the copyright to your article

Submit your next manuscript at $>$ springeropen.com 